\title{
NUMERICAL SIMULATION OF PARTICLE MOVEMENT IN CELLULAR FLOWS UNDER THE INFLUENCE OF MAGNETIC FORCES
}

\author{
Ravnik, J.*; Hribersek, M. ${ }^{*}$; Vogel, F. ${ }^{* *} \&$ Steinmann, P. ${ }^{* *}$ \\ *Faculty of Mechanical Engineering, University of Maribor, Smetanova 17, 2000 Maribor, Slovenia \\ ${ }^{* *}$ University of Erlangen-Nuremberg, Chair of Applied Mechanics, Egerlandstrasse 5, \\ 91058 Erlangen, Germany \\ E-Mail: jure.ravnik@um.si
}

\begin{abstract}
A numerical model of particle motion in fluid flow under the influence of hydrodynamic and magnetic forces is presented. The Lagrangian particle tracking algorithm was developed being capable of simulating dilute suspensions of particles in viscous flows where gravity, buoyancy, drag, pressure gradient, added mass and magnetophoretic forces are taken into account. The method is used to study the behaviour of magnetite particles in a periodic cellular flow field under the influence of a magnetic field produced by electric wires placed in cell centres. For such a flow field it is known that particles in steady state merge into individual trajectories. The influence of the magnetic field on the particle trajectories is examined and an exponential model for the time evolution of the fraction of adhered particles to the electric wires is proposed. Three particle Stokes number values are considered: 0.01 , 0.1 and 1 . The existence of a critical magnetic pressure coefficient was found, at which all particles end up to be adhered to the wires. The critical magnetic pressure coefficient was found to be proportional to the Stokes number. For sub-critical magnetic pressure coefficient, the particle trajectories are significantly altered by the magnetic field, both in their shape and in their number. Furthermore, in the sub-critical regime, the minimal distance of particles to the cell centres is larger for particles with smaller Stokes numbers.
\end{abstract}

(Received in October 2013, accepted in March 2014. This paper was with the authors 2 months for 1 revision.)

Key Words: Dispersed Two Phase Flow, Lagrangian Particle Tracking, Magnetic Force, Hydrodynamic Forces, Cellular Flow

\section{INTRODUCTION}

Nano and micro particles with magnetic properties are increasingly gaining interest in several fields of science, like life sciences, natural sciences, and medicine or engineering sciences. The dimensions of magnetic nanoparticles are smaller than or comparable to the size of cells, viruses or proteins, enabling them to interact with biological agents directly and thereby providing a controllable means of tagging and addressing specific cells. In the case of development of novel actuators, e.g. for artificial muscles for robot locomotion, novel magneto-sensitive polymeric materials are one of the current research topics. Although in their final form these materials are elastic solids with a rubber-like matrix that is filled with magneto-active particles, the production process consists of mixing liquid components with magneto-active particles. Due to magnetic properties, the movement of the particles may be manipulated with an external magnetic field gradient, Pankhurst et al. [1]. Iron oxides, i.e. magnetite and maghemite, are the most frequently used because of their generally suitable magnetic properties and biological compatibility. The design and control of magnetic fields is an active research field (Lipus et al. [2]), as well as the effect of magnetic forces on particles in flows. This was considered by Yang et al. [3], who studied motions of magnetic nanospheres under the magnetic field in the rectangular microchannel, as well as by Zolgharni 
et al. [4] who performed a numerical design study of chaotic mixing of magnetic particles in a microfluidic bio-separator.

Efficient algorithms for Lagrangian particle tracking in fluid flow are an ongoing research topic (Cohen Stuart et al. [5]) simulating particles in laminar and turbulent flows (Marchioli et al. [6]). In our work, as a starting point, the earlier work by Ravnik et al. [7] was chosen, where a BEM-FEM algorithm for a 2D flow simulation was coupled with an explicit Lagrangian particle tracking algorithm. The algorithm was extended to a 3D geometry in Ravnik et al. [8]. In order to realistically capture the particle response to fluid flow structures, a one-way coupling approach was implemented. The particles were moving due to the action of gravity, buoyancy, drag, pressure gradient and added mass forces. Since numerical simulation of dilute suspensions is the main target of this work, the coupling between the two phases is a one-way action of the fluid on the particles. The derived method can be extended to capture also flows with particles that have a high magnetic susceptibility, in the case when the fluid flows through the area of a non-uniform external magnetostatic field, which gives rise to a magnetophoretic force. The force is proportional to the magnetic field gradient acting on the magnetic moment of the particle.

Gravitational settling and suspension of particles has been under intense examination in the past as it is important to many areas of engineering and environmental sciences. Cellular flow regimes frequently occur in natural convection flows, resulting from thermal instabilities, and are thus important in separation engineering as well as in thermal engineering. Therefore, stochastic random flow fields as well as periodic cellular fields were already used as model problems. Maxey and Corrsin [9] computed statistics for the motion of small particles settling under gravity in an ensemble of randomly oriented, periodic, two dimensional cellular flow fields that are steady in time. They found that inertia, although weak, eventually causes all particles to settle out at a rate that over most parametric ranges is higher than in a still fluid. Particles with small free fall velocity and weak inertia show a strong tendency to collect along isolated paths. Maxey [10] studied the motion of small spherical particles in a cellular flow field. The study revealed the possibility that the particles remain suspended in the flow field, a phenomenon which was observed while studying plankton movement. Rubin et al. [11] presented a proof that given a dilute concentration of aerosol particles in an infinite, periodic, cellular flow field, arbitrarily small inertial effects are sufficient to induce almost all particles to settle. They showed that settling particles approach a finite number of attracting periodic paths. The structure of the set of attracting paths, including the nature of possible bifurcations of these paths and the resulting stability changes, is examined via a symmetric one-dimensional map derived from the flow. Influence of unsteady cellular flow on the gravitational settling rate of heavy spherical particles was studied by Fung [12]. The change in settling velocity of inertial particles in cellular flow was studied by Chan and Fung [13]. The problem was revisited recently by Marchioli et al. [14], who provided additional results. For the case of small naturally buoyant particles, segregation of particles in cellular flows was studied by Tallapragada and Ross [15]. Thus, particle movement patterns for the whole range of density ratios and Stokes numbers are available.

This paper analyses the effect of the magnetic force acting on the particles in a cellular flow field, identifying how the magnetic force alters the balance of gravity and hydrodynamic forces and what effect does the magnetic field have on the steady state particle trajectories. A special attention is paid to the influence of the magnetic field on the collection efficiency of electric wires. The cellular flow has been chosen because it is a model for a vortical flow, in which influence of the magnetic field can be studied. The flow is assumed to be Newtonian and the fluid not electrically conducting, magneto-hydrodynamics effects are not taken into account. 


\section{MAGNETIC FORCE ON A SINGLE PARTICLE}

The iron based particles are ferromagnetic in their monolithic form, but when they have diameters in the range of nanometers they behave in a similar manner as paramagnets. For larger, micrometer range particles, a slight deviation from paramagnetic behaviour in form of magnetic memory can be expected. Mixing of magnetic particles with an appropriate polymer to form a magnetic bead can help solve this problem.

In the case of a particle with a magnetic moment in a nonuniform external magnetic field, the Kelvin body force acts on a single particle. The established model for the magnetic force on a single particle reads as:

$$
\vec{F}_{m}=\frac{1}{2} \mu_{0}\left[\chi_{p}-\chi_{f}\right] V_{p} \vec{\nabla}(\vec{H} \cdot \vec{H})
$$

with $\left[\chi_{p}-\chi_{f}\right]$ the magnetic susceptibility difference between the particle and the fluid, and $V_{p}$ the particle volume. The point value of the local magnetic field strength $\vec{H}$ at the location of a particle can be used since the distance between the poles of the particle is much smaller than the distance between the poles of the external source. Also, with $\vec{H}$ the magnetic field strength, the following expression $\vec{\nabla}(\vec{H} \cdot \vec{H})=2(\vec{H} \cdot \vec{\nabla}) \vec{H}$ is valid. Therefore, the force on a particle is proportional to the strength of magnetic field and the field gradient. Thus the final expression for the Kelvin body force is:

$$
\vec{F}_{m}=\mu_{0} V_{p}(\vec{H} \cdot \vec{\nabla}) \vec{M}_{r},
$$

with the relative magnetization being defined as $\vec{M}_{r}=\left[\chi_{p}-\chi_{f}\right] \vec{H}$. When two or more particles interact, additional attractive or repulsive forces are present, depending on the dipole orientation of the particles.

\section{LAGRANGIAN PARTICLE TRACKING}

Let us consider spherical particles of diameter $d_{p}$, mass $m_{p}$ and density $\rho_{p}$. The mass of fluid encompassing the same volume as the particle is denoted by $m_{f}$. The equation of motion for small rigid spheres was proposed by Maxey and Riley [16]. Neglecting the aerodynamic lift, time history effects and second order terms, due to small particle size, we may write:

$$
\begin{aligned}
& m_{p} \frac{d \vec{v}}{d t}=\left(m_{p}-m_{f}\right) \vec{g}+m_{f} \frac{D \vec{u}}{D t}-\frac{1}{2} m_{f}\left(\frac{d \vec{v}}{d t}-\frac{d \vec{u}}{d t}\right)- \\
& -3 \pi d_{p} \rho_{f} v(\vec{v}-\vec{u})\left(1+0.15 \operatorname{Re}_{p}^{0.687}\right)+\mu_{0} V_{p}(\vec{H} \cdot \vec{\nabla}) \vec{M}_{r} .
\end{aligned}
$$

Here $\vec{v}$ is the velocity of the particle and $\vec{u}$ is the fluid velocity. The terms included in the equation are gravity, buoyancy, pressure gradient term, added mass term, drag (skin friction and form drag) and the magnetic force term. The Stokes drag term has been corrected using the heavy particle correction term, which is valid for particle Reynolds numbers up to $R e_{p}=\frac{d_{p}}{v}|\vec{u}-\vec{v}|<1000$. Here, $d / d t=\partial / \partial t+(\vec{v} \cdot \vec{\nabla})$ stands for the time derivative following the particle and $D / D t=\partial / \partial t+(\vec{u} \cdot \vec{\nabla})$ is the time derivative following the fluid element.

Eq. (3) is rewritten in a non-dimensional form with $H_{0}, u_{0}$ and $L$ being the characteristic magnetic field strength, the characteristic fluid velocity scale and the characteristic problem length scale, respectively. With $\vec{H} \rightarrow \vec{H} / H_{0} \vec{u} \rightarrow \vec{u} / u_{0}, \vec{v} \rightarrow \vec{v} / u_{0}$ and $t \rightarrow t u_{0} / L$ this renders eventually to: 


$$
\begin{aligned}
\vec{a}=\frac{d \vec{v}}{d t}= & \frac{A}{S t}\left\{\vec{v}_{s}+(\vec{u}-\vec{v})\left(1+0.15 \operatorname{Re}_{p}^{0.687}\right)\right\}+\frac{3}{2} R \frac{\partial \vec{u}}{\partial t}+ \\
& +R\left\{\left(\vec{u}+\frac{1}{2} \vec{v}\right) \cdot \vec{\nabla}\right\} \vec{u}+R C_{p m}(\vec{H} \cdot \vec{\nabla}) \vec{M}_{r},
\end{aligned}
$$

where the Stokes number is defined as:

$$
S t=\frac{\rho_{p} d_{p}^{2} u_{0}}{\rho_{f} 18 v L}
$$

the settling velocity is:

$$
\vec{v}_{s}=\frac{d_{p}^{2}}{18 v u_{0}}\left(\frac{\rho_{p}}{\rho_{f}}-1\right) \vec{g},
$$

the magnetic pressure coefficient (Sobral and Cunha [17]), which represents the importance of the magnetic pressure with respect to the dynamic pressure of the fluid phase, is:

$$
C_{p m}=\frac{\mu_{0} H_{0}^{2}}{u_{0}^{2} \rho_{f}}
$$

and the parameters $R$ and $A$ are:

$$
R=\frac{\rho_{f}}{\rho_{p}+\frac{1}{2} \rho_{f}}, \quad A=\frac{\rho_{p}}{\rho_{p}+\frac{1}{2} \rho_{f}} .
$$

In the case of very light particles $\left(\rho_{p}<<\rho_{f}\right)$ the parameters $R$ and $A$ tend to $R \rightarrow 2, A \rightarrow 0$. In the aerosol limit $\left(\rho_{p}>>\rho_{f}\right)$, the parameters reach their limits as $R \rightarrow 0, A \rightarrow 1$, rendering the pressure gradient and added mass terms negligible. The term $0.15 R e_{p}^{0.687}$ is the heavy particle correction term, which is included in the equation only in the aerosol limit. For fluid particles $\left(\rho_{p}=\rho_{f}\right)$, we have $R=A=2 / 3$.

With the acceleration of the particle given in eq. (4) we may solve the particle equation of motion by employing the $4^{\text {th }}$ order Runge-Kutta method. We integrate the following six equations simultaneously:

$$
\frac{d x}{d t}=v_{x}, \quad \frac{d v_{x}}{d t}=a_{x}, \quad \frac{d y}{d t}=v_{y}, \quad \frac{d v_{y}}{d t}=a_{y}, \quad \frac{d z}{d t}=v_{z}, \quad \frac{d v_{z}}{d t}=a_{z}
$$

The unknowns are the particle location $(x, y, z)$ and particle velocity $\left(v_{x}, v_{y}, v_{z}\right)$. The initial particle location and velocity must be known. In order to calculate the acceleration contributions on the right hand side, the velocity of the fluid, $\vec{u}$, has to be calculated at the location of the particle. The solution algorithm described in detail in Ravnik et al. [7] is used.

\section{CELLULAR FLOW IN MAGNETIC FIELD}

Cellular flow is an infinite field of two dimensional steady vortices. Let the domain be given by $\Omega=[0, L] \times[0, L]$ with periodic boundary conditions. In this case the cellular flow velocity $\vec{u}$ and vorticity $\vec{\omega}$ fields are:

$$
\vec{u}=u_{0}\left\{\begin{array}{c}
\sin \frac{2 \pi x}{L} \cos \frac{2 \pi z}{L} \\
0 \\
\cos \frac{2 \pi x}{L} \sin \frac{2 \pi z}{L}
\end{array}\right\}, \quad \vec{\omega}=\frac{u_{0}}{L}\left\{\begin{array}{c}
0 \\
\pi \sin \frac{2 \pi x}{L} \sin \frac{2 \pi z}{L} \\
0
\end{array}\right\},
$$


with gravity acting in the negative $z$-direction; $\vec{g}=\left(0,0,-9.81 \mathrm{~m} / \mathrm{s}^{2}\right)$. The flow field is periodic, thus particles exiting the domain on one side are inserted back into the domain on the opposite side.

According to the study of Hong et al. [18], magnetite nanoparticles have a density of $\rho_{p}=5180 \mathrm{~kg} / \mathrm{m}^{3}$ and a magnetic susceptibility of $\chi_{p}=0.41$. We will consider the behaviour of these magnetite nanoparticles suspended into a cellular flow $\left(L=1 \mathrm{~m}, u_{0}=1.01 \mathrm{~m} / \mathrm{s}\right)$ of water $\left(\rho_{f}=998 \mathrm{~kg} / \mathrm{m}^{3}, v=1.01 \mathrm{~mm}^{2} / \mathrm{s}\right)$. This makes the non-dimensional time unit $t=\frac{L}{u_{0}}=0.99 \mathrm{~s}$. Magnetic susceptibility of water is $\chi_{f}=-9 \cdot 10^{-6}$. The density ratio yields $R=0.176$ and $A=0.912$ according to eq. (8). We consider particles of three different diameters, which have Stokes numbers of $S t=0.01, S t=0.1$ and $S t=1$ and settling velocities of $0.0776,0.776$ and 7.76 given by eq. (6), respectively.

Let us consider a magnetic field produced by a long thin straight electrical wire running in the $y$-direction. The magnetic field strength $\vec{H}$ of an arbitrary electrical conduit may be calculated using the Biot-Savart equation:

$$
\vec{H}=\frac{I}{4 \pi} \int_{-\infty}^{+\infty} \frac{\vec{r} \times \overrightarrow{d l}}{r^{3}}=\frac{I}{2 \pi r}\left(\begin{array}{c}
-\sin \alpha \\
0 \\
\cos \alpha
\end{array}\right)
$$

where $I$ denotes the electric current, $r$ is the distance from an observation point to the wire and $\alpha$ is the angle between the observation point and the $x$-axis. Using this result, the magnetic force (2) may be evaluated for the case of infinite straight wire conduit as:

$$
\vec{F}_{m}=-\mu_{0}\left(\chi_{p}-\chi_{f}\right) V_{p} \frac{I^{2}}{4 \pi^{2} r^{3}}\left(\begin{array}{c}
\cos \alpha \\
0 \\
\sin \alpha
\end{array}\right)
$$

We consider thin electric wires placed in the centre of each vortex and assume that the wires do not disturb the flow field. The electric current runs in the opposite direction in the neighbouring wires. The resulting magnetic field is calculated by summation of the contributions of each wire. Considering (12), we may write the nondimensional contribution of the magnetic forces to the particle acceleration as:

$$
\vec{a}_{m}=-C_{p m} \frac{\rho_{f}}{\rho_{p}}\left(\chi_{p}-\chi_{f}\right) \sum_{i=1}^{\infty} \frac{1}{r_{i}^{3}}\left(\begin{array}{c}
\cos \alpha_{i} \\
0 \\
\sin \alpha_{i}
\end{array}\right),
$$

with $i$ denoting the $i^{\text {th }}$ wire, magnetic pressure coefficient being $C_{p m}=\frac{\mu_{0}}{u_{0}^{2} \rho_{f}}\left(\frac{I}{2 \pi L}\right)^{2}$, characteristic magnetic flux density is $B_{0}=\frac{\mu_{0} I}{2 \pi L}$ and $r_{i}$ represents the nondimensional distance from the particle location to the $i^{\text {th }}$ wire.

In the simulation $2 \cdot 10^{4}$ particles were included. Initially, the particles were distributed randomly in the cellular flow field having velocities equal to the fluid velocity. It has been shown (Fung [12]) that initial position and initial velocity of the particles have no influence on the steady state particle trajectories. However, in our case, when magnetic forces are present, the number of particles which adhere to the wires depends on the initial position and velocity due to the fact that the magnetic force depends on the distance from the particles to the wires. Thus, the fraction of particles that adhere to the wires in steady state is different for 
each different initial distribution of particles, while the steady state particle trajectories are unaffected by the initial conditions.

A time step of $10^{-4}$ was used to advance the particles for up to $1.1110^{7}$ time steps until the total nondimensional time of 1100.0 was reached. The time span was long enough for the particle motion to reach steady state, i.e. the particles found stationary paths through the flow field repeating them over and over again due to the periodic boundary conditions. Shorter total simulation times were used in cases when steady state was reached earlier. Several electric currents were used, which gave magnetic flux densities ranging between $B_{0}=1 \mathrm{mT}$ to $B_{0}=30 \mathrm{mT}$. The corresponding magnetic pressure coefficients were $C_{p m}=7.89 \cdot 10^{-4}$ and $C_{p m}=0.5$, respectively.

As the simulation progresses, the particle's movement is affected by gravity, vertical flow structure and the magnetic force. The magnetic force moves the particles towards the wires. We assume the wires to have a nondimensional diameter of 0.01 . If the particle hits a wire, it is assumed to adhere to the wire. Since the magnetic force decreases very rapidly away from the wire $\left(\propto 1 / r^{3}\right)$, the number of particles attached to the wire depends on the magnetic flux density.

\section{RESULTS}

\subsection{Case of $S t=0.1$ and $C_{p m}=0.08$}

Let us examine the result of the simulation with particles having $S t=0.1$ in the case where the electric current in the wires produces a magnetic field of $C_{p m}=0.08$. At the beginning of the simulation, particles are randomly distributed. As time progresses, gravity forces the particles in a downward direction. Flow induced drag accounts for the vortical structure and the magnetic forces tend to push the particles towards the wires. During the simulation some particles are adhered to the wires while others manage to avoid the influence of the magnetic forces and find their way through the flow field. Their movement is then governed mainly by gravity and drag. Steady state is reached after a long time. In the steady regime, particles repeatedly travel through the flow field always along the same paths due to periodic boundary conditions. The same phenomenon was observed for the flow field without magnetic forces.

In order to assess the influence of the magnetic force, we calculated the forces acting on each particle at each time step. For each position in the flow domain, the magnitude of forces on each particle crossing that position were averaged and finally divided be the sum of all forces, thus calculating the relative effect of active forces. As expected from eq. (13), the magnetic force dominates all other forces in the vicinity of the wires. Drag is the predominant force between the vortices, where particles travel downwards. Gravity dominates in areas where particles travel sideways, i.e. perpendicularly to the direction of gravity. The contributions of added mass and pressure correction terms are small, reaching up to maximally $10 \%$ of the total force acting on particles.

Choosing a single particle, a time trace of forces acting on the particle is presented in Fig. 1. Since gravitational and buoyancy contributions to the total force do not change with time, we present the forces relative to this value. The graph presents only steady state, therefore the first $10^{5}$ time steps were omitted. We can readily observe the periodic nature of the forces. As the particle repeatedly travels through the periodic flow structure, it encounters the same forces again and again. Since the particle in question found its way through the flow field without being pulled by magnetic force towards the wire, we observe that the magnetic force along most of its path is at least an order of magnitude smaller than gravity and two orders of magnitude smaller than drag. 

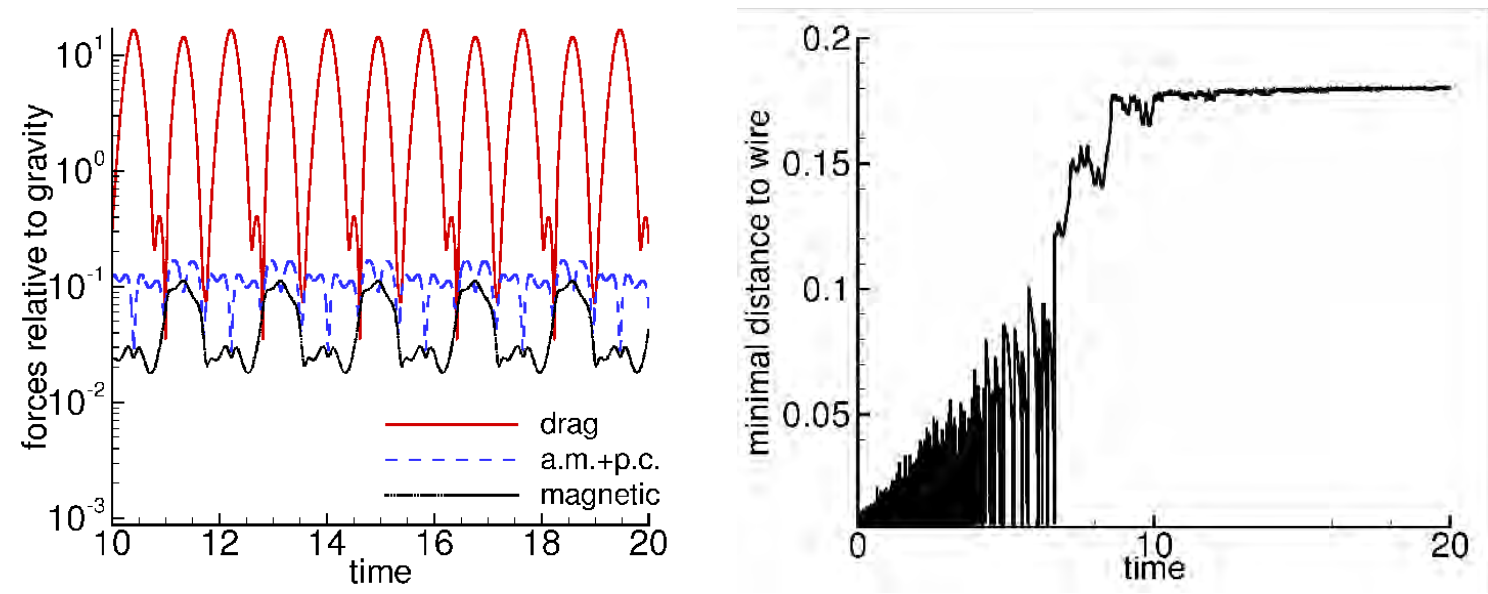

Figure 1: Temporal trace of forces acting on a single particle (left) and temporal development of the distance between the wire and the closest particle (right); case of $S t=0.1, C_{p m}=0.08$.

In each time step during the simulation we calculated the distance from the wires to the nearest particle. The result is shown in the right hand side of Fig. 1. During the initial phase of the simulation, a lot of particles adhere to the wires making the minimal distance nearly zero. As time progresses, less and less particles are captured by the magnetic field and finally all of the remaining particles manage to avoid the wires. Particle motion is then governed mainly by gravity and drag, thus the particles are thrown out of the vortices, which makes the minimal distance to wires (which are located in centres of vortices) larger. Finally, when steady state is reached, the minimal distance converges to a constant value.

Next, we examine the number of particles adhered to the electric wires by expressing this number as a fraction of all particles $S$. We observe that the fraction of adhered particles increases rapidly in the beginning of simulation. The flux of particles to the wires decreases as the simulation progresses. It stops entirely when steady state is reached. The behaviour seems to be governed by an exponential function. It is well known that exponential behaviour is found in cases where the flux (of, for example, heat or mass) is proportional to the amount of heat or mass. This situation occurs in our simulation as well - the number of particles adhering to the wires is proportional to the number of particles in the vicinity of the wires. Denoting the fraction of adhered particle in steady state by $S_{\infty}$, we performed a least squares fit of the following model:

$$
S=S_{\infty}\left(1-e^{-\alpha t}\right),
$$

which for the case $S t=0.1$ and $C_{p m}=0.08$ yielded $S_{\infty}=0.22, \alpha=3.02$ and $\beta=0.77$.

\subsection{Influence of magnetic field strength}

Now, let us consider the effect of the magnetic field. Keeping the value of $S t=0.1$ constant for the particles, we varied the magnetic pressure coefficient up to $C_{p m}=0.5$. Remembering that the magnetic force scales with a square of $B_{0}$ and linearly with $C_{p m}$, we found that for a large magnetic pressure coefficient all particles adhere to the wires. Let the minimal magnetic pressure coefficient, which causes all particles to adhere to the wires, be called the critical magnetic pressure coefficient. Above the critical magnetic pressure coefficient the magnetic force is strong enough to pull all particles towards the wires regardless of the gravity and drag caused by the vortical flow structure. For $C_{p m} \geq 0.5$ and $S t=0.1$ all particles end up adhered to wires at steady state. The time development of fraction of adhered particles is shown in Fig. 2 (left), where a comparison between different values for $C_{p m}$ can be observed. In all cases the 
exponential model (14) describes this behaviour very well, with the fitting constants given in Table I.
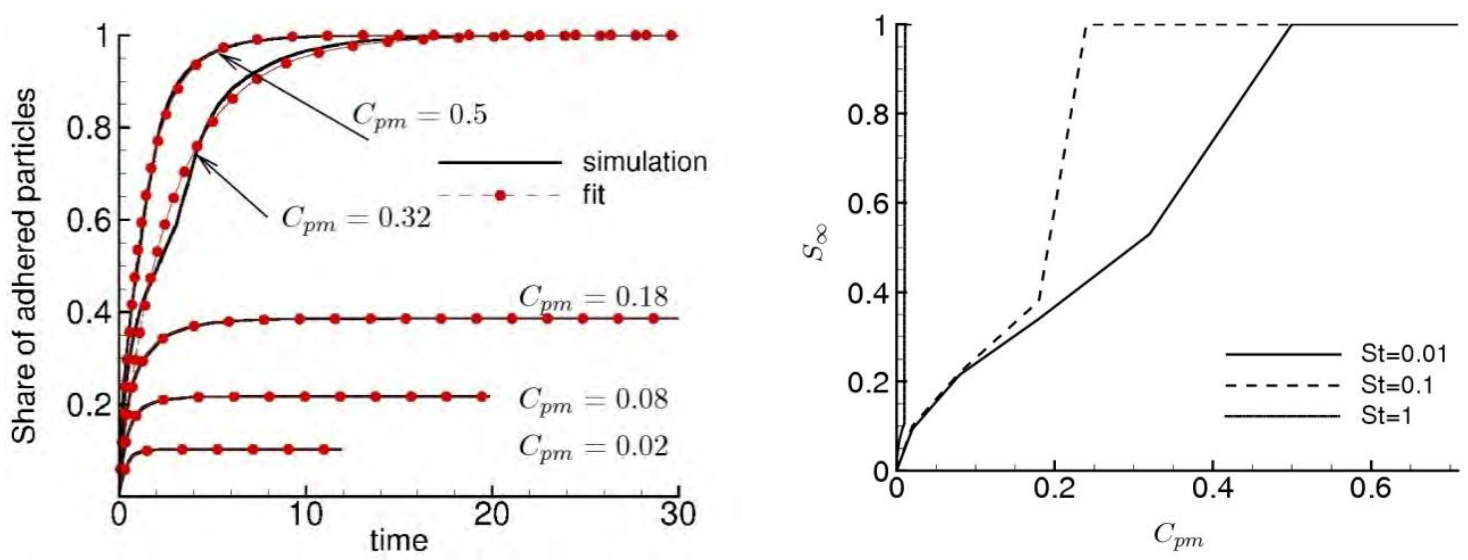

Figure 2: Time development of fraction of particles attached to the wires for different magnetic field strengths $(S t=0.1)$ - left; Fraction of adhered particles $S_{\infty}$ depends highly on the magnetic pressure number $C_{p m}$ - right.

Table I: Minimal distance to wire $d_{\min }$, fraction of adhered particles in steady state $S_{\infty}$, exponential fit of the number of adhered particles in steady flow regime, $\alpha, \beta$ and time $t_{99}$ at which $0.99 S_{\infty}$ of particles are adhered to the wires.

\begin{tabular}{|c|c|c|c|c|c|c|}
\hline$B_{0}[\mathrm{mT}]$ & $C_{p m}$ & $d_{\text {min }}$ & $S_{\infty}$ & $\alpha$ & $\beta$ & $t_{99}$ \\
\hline & \multicolumn{7}{|c|}{$S t=0.1$} \\
\hline 0 & 0 & 0.239 & 0.0 & & & \\
\hline 5 & 0.02 & 0.239 & 0.093 & 0.85 & 0.67 & 12.5 \\
\hline 10 & 0.08 & 0.2385 & 0.215 & 0.71 & 0.63 & 19.2 \\
\hline 15 & 0.18 & 0.237 & 0.34 & 0.58 & 0.62 & 28.6 \\
\hline 20 & 0.32 & 0.232 & 0.53 & 0.45 & 0.60 & 48.6 \\
\hline 25 & 0.5 & 0.0 & 1.0 & 0.26 & 0.42 & $912^{*}$ \\
\hline 30 & 0.71 & 0.0 & 1.0 & 0.27 & 0.66 & 72.8 \\
\hline \multicolumn{7}{|c|}{$S t=0.1$} \\
\hline 0 & 0 & 0.20778 & 0.0 & & & \\
\hline 5 & 0.02 & 0.20765 & 0.10 & 2.91 & 0.89 & 1.97 \\
\hline 10 & 0.08 & 0.181 & 0.22 & 1.77 & 0.77 & 3.46 \\
\hline 15 & 0.18 & 0.169 & 0.38 & 1.22 & 0.69 & 6.89 \\
\hline 17.5 & 0.24 & 0.0 & 1.0 & 0.19 & 0.85 & $41.8^{*}$ \\
\hline 20 & 0.32 & 0.0 & 1.0 & 0.41 & 0.88 & 15.8 \\
\hline 25 & 0.5 & 0.0 & 1.0 & 0.77 & 0.90 & 7.33 \\
\hline \multicolumn{7}{|c|}{$S t=1.0$} \\
\hline 0 & 0 & 0.0437 & 0.0 & & & \\
\hline 1 & 0.0008 & 0.0433 & 0.033 & 2.81 & 1.12 & 1.55 \\
\hline 2 & 0.0032 & 0.0425 & 0.065 & 2.98 & 1.14 & 1.46 \\
\hline 3 & 0.0071 & 0.0415 & 0.092 & 3.04 & 1.11 & 1.45 \\
\hline 3.5 & 0.0097 & 0.03266 & 0.104 & 2.96 & 1.07 & 1.51 \\
\hline 3.75 & 0.0111 & 0.0 & 1.0 & 0.073 & 0.8 & $177^{*}$ \\
\hline 4 & 0.0126 & 0.0 & 1.0 & 0.15 & 1.47 & 10.4 \\
\hline 5 & 0.0197 & 0.0 & 1.0 & 0.25 & 1.46 & 7.32 \\
\hline
\end{tabular}

(* model fit is poor close to the critical magnetic pressure coefficient) 
In Fig. 3 we compare steady state distribution of $S t=0.1$ particles for different magnetic pressure coefficients. When the magnetic field is weak $\left(C_{p m}=0.02\right)$, the particles merge into an isolated trajectory, independent of the initial position and initial velocity. The minimal distance to vortex centres, which is listed in Table I, is only slightly smaller than in the case of no magnetic field. However, when the magnetic pressure coefficient is increased, we observe additional trajectories and the minimal distance to the vortex centres is significantly reduced. The magnetic force bends the trajectories and allows additional trajectories to be established.

In Table I, the steady state minimal distance between the wire and the nearest particle is shown for different Stokes numbers and magnetic pressure coefficients. For low magnetic fields and low Stokes numbers the value approaches the theoretically largest value of 0.25 . As the magnetic pressure coefficient increases, the particle paths are moved closer to the wires and closer to the vortex cores and thus the minimal distance decreases. The process continues up to the critical magnetic pressure coefficient, when all particles are adhered to the wires.
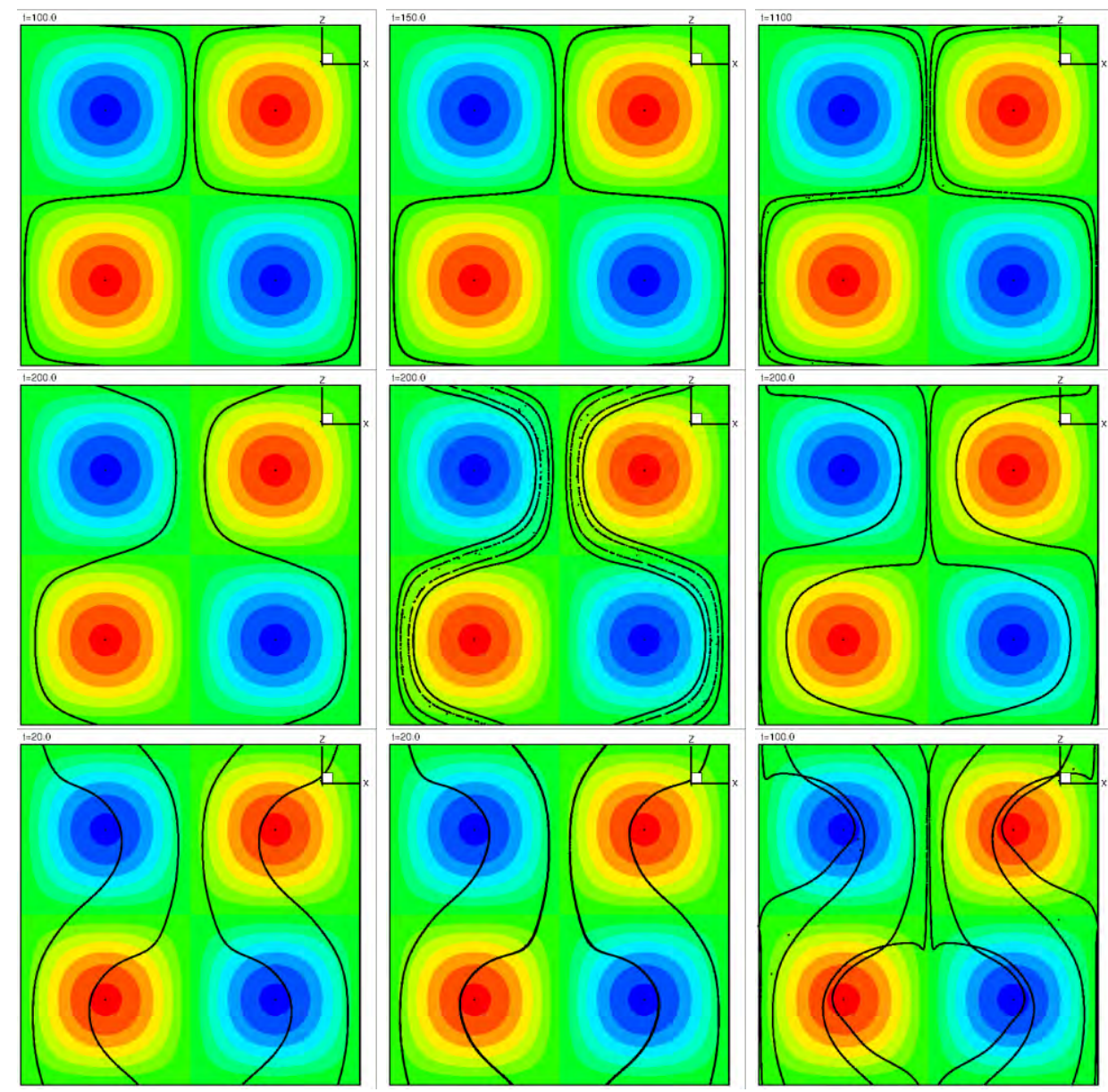

Figure 3: Steady distribution of particle positions;

top row: $S t=0.01$, left $C_{p m}=0.02$, centre $C_{p m}=0.18$ and right $C_{p m}=0.32$;

middle row: $S t=0.1$, left $C_{p m}=0.02$, centre $C_{p m}=0.08$ and right $C_{p m}=0.18$;

bottom row: $S t=1.0$, left $C_{p m}=0.0032$, centre $C_{p m}=0.0071$ and right $C_{p m}=0.0097$.

\subsection{Influence of Stokes number}

Next, we considered the influence of the Stokes number. At low Stokes number $(S t=0.01)$, the drag force dominates the behaviour of the particles. Particles follow the fluid flow almost ideally making many revolutions in vortices before they are either adhered to the wires or thrown out of the vortices. At high Stokes number $(S t=1)$, the influence of the flow on the 
particle movement is small and particle behaviour is governed mainly by gravity and magnetic forces. Thus, at high St a weak magnetic field is able to adhere a large number of particles to the wires, whereas at low $S t$ a much stronger magnetic field is required in order to achieve the same effect. This can be observed by examining the fractions of adhered particles $S_{\infty}$ in steady regime, listed in Table I.

Consider now the steady state distributions for $S t=0.01$ particles, shown in Fig. 3. With $C_{p m}=0.5$ all of the particles travel around the vortices, circling the wires. Some make many revolutions, but eventually after a long time $(t=215)$ each and every one of them is pulled towards the wires by the magnetic force and end up adhered to the wires. If the magnetic pressure coefficient is increased even further to $C_{p m}=0.71$, the particles are captured by the wires faster, only $t=100$ is needed for all particles to be adhered. This is noticeable when comparing the fitted $\beta$ value in Table I. The $\beta$ is smaller when particles are adhered faster.

Particles having a high Stokes number find their way through the vortices, their path leading mostly in the direction of gravity. The drag force does not dominate the behaviour, but rather only mildly alters the motion. As the electric current is injected into the wires the resulting magnetic field of $C_{p m} \geq 0.0111$ is already strong enough to adhere all of the particles to the wires. A magnetic field of $C_{p m} \leq 0.0071$ on the other hand only slightly alters the path of the particles. The minimal distance from particles to the wires (Table I) increases only slightly when the magnetic pressure coefficient is increased. Only a small percentage of particles end up adhered to the wires, as can be seen by examining $S_{\infty}$ in Table I. When the magnetic pressure coefficient is increased from $C_{p m}=0.0071$ to $C_{p m}=0.0126$, the magnetic force is strong enough such that all particles are adhered to the wires.

\subsection{Critical magnetic pressure coefficient}

Comparing the critical magnetic pressure coefficient that is required to adhere all particles to the wires, we found that it decreases with increasing Stokes number. At $S t=0.01$, a magnetic pressure coefficient of $C_{p m}=0.71$ is required, while at $S t=0.1, C_{p m}=0.32$ is needed and finally at $S t=1.0$ only $C_{p m}=0.0126$ is necessary. When the magnetic pressure coefficient is near the critical value, the increase of the fraction of adhered particles with time does not follow closely the model proposed in eq. (14). The model fit is poor compared to other magnetic pressure coefficients, where the model closely fits the data. A certain lack of accuracy can be observed in Fig. 2 for $S t=0.1$ and $C_{p m}=0.32$. This observation holds for all considered Stokes numbers. The reason for this phenomenon can be explained in the following way: at the beginning of simulation, particles close to the wires are adhered quickly. Their behaviour follows closely the proposed exponential model. Later, since the magnetic pressure coefficient is close to the critical value, other particles are also drawn towards the wires, but since the magnetic force is almost balanced with drag and gravity, this procedure takes longer and is not in accordance with the exponential model. The accuracy of the exponential fit deteriorates at low Stokes numbers, where particles make many revolutions before adhering to the wires.

\subsection{Minimal distance to the wires}

Examining the minimal distance from particles to wires in Table I, we observe that the distance is largest for small Stokes number, while particles with large Stokes number are able to travel closer to the wires. At low Stokes number, drag plays an important role in determining the trajectory of the particles, forcing them out of the vortices. When a subcritical magnetic pressure coefficient is applied, the particles are only slightly pulled towards the centres of the wire. On the other hand, particles with high Stokes number are able to travel 
through the vortices, since gravity dominates over drag. Here, already a small magnetic force pulls them even closer to the wires.

\subsection{Fraction of adhered particles}

In Fig. 2 (right) we examine the dependence of the steady state value of the fraction of adhered particles $S_{\infty}$ on the magnetic pressure coefficient. For all Stokes numbers we observe an existence of a critical magnetic pressure coefficient, which causes all of the particles to adhere to the wires. For large Stokes numbers the critical magnetic pressure coefficient is smaller than in the case of smaller Stokes numbers. This can be explained by the fact that vortical flow has less effect on the particles in case of large Stokes numbers and particles are able to reach the vicinity of the wires. For small Stokes numbers, the particles tend to be transported out of the vortices by the flow and thus away from the area where magnetic force is large. It can also be observed that the dependence of the fraction of adhered particles on the magnetic pressure coefficient has a large gradient close to the critical magnetic pressure coefficient. The high gradient, i.e. the jump in $S_{\infty}$ when approaching the critical magnetic pressure coefficient, is largest in the case of low Stokes numbers. For example, for $C_{p m}=0.0097$ only about $10 \%$ of the particles are adhered to the wires, while at $C_{p m}=0.0111$ all of the particles are adhered to the wires. This gradient decreases when the Stokes number is increased.

\section{CONCLUDING REMARKS}

Cellular flow is a model, which enables study of particle transport phenomena. As a well defined test problem, it is frequently used in detailed studies of particle motion in fluid flow. In this study we extended the test problem by applying a magnetic field by placing electric wires in cell centres and studying the behaviour of magnetite nanoparticles in water. Initially the particles were randomly distributed in the flow field. Lagrangian particle tracking method was used to simulate dilute suspension of particles taking into account gravity, buoyancy, drag, pressure gradient, added mass and magnetophoretic forces.

We observed an existence of a critical magnetic pressure coefficient, which causes all of the particles to adhere to the wires. The critical magnetic pressure coefficient increases with increasing particle Stokes number. Looking at the time history of the fraction of adhered particles we proposed an exponential model, which describes the phenomena very well. The proposed model can be used to predict the time needed for all particles to adhere at a chosen magnetic pressure coefficient. Also, the influence of the magnetic pressure coefficient on the steady state particle trajectory was examined. We found that under a magnetic field influence particles in steady state merge into isolated trajectories, similarly as in the non-magnetic case. However, the trajectories are significantly altered; particle paths are moved closer to the source of the magnetic field and even the number of trajectories is changed.

The performed computational study can therefore help to better understand the interplay of gravitational, hydrodynamic and magnetic forces acting on particles suspended in cellular flows.

\section{REFERENCES}

[1] Pankhurst, Q. A.; Connolly, J.; Jones, S. K.; Dobson, J. (2003). Applications of magnetic nanoparticles in biomedicine, Journal of Physics D: Applied Physics, Vol. 36, No. 13, 167-181, doi: $10.1088 / 0022-3727 / 36 / 13 / 201$ 
[2] Lipus, L. C.; Acko, B.; Hamler, A. (2012) Magnetic device simulation modelling and optimisation for scale control, International Journal of Simulation Modelling, Vol. 11, No. 3, 141-149, doi:10.2507/IJSIMM11(3)3.205

[3] Yang, J.; Park, J.; Lee, J.; Cha, B.; Song, Y.; Yoon, H.-G.; Huh, Y.-M.; Haam, S. (2007). Motions of magnetic nanosphere under the magnetic field in the rectangular microchannel, Journal of Magnetism and Magnetic Materials, Vol. 317, No. 1-2, 34-40, doi:10.1016/j.jmmm.2007.04.008

[4] Zolgharni, M.; Azimi, S. M.; Bahmanyar, M. R.; Balachandran, W. (2007). A numerical design study of chaotic mixing of magnetic particles in a microfluidic bio-separator, Microfluidics and Nanofluidics, Vol. 3, No. 6, 677-687, doi:10.1007/s10404-007-0160-9

[5] Stuart, D. C. C.; Kleijn, C. R.; Kenjeres, S. (2011). An efficient and robust method for Lagrangian magnetic particle tracking in fluid flow simulations on unstructured grids, Computers \& Fluids, Vol. 40, No. 1, 188-194, doi:10.1016/j.compfluid.2010.09.001

[6] Marchioli, C.; Fantoni, M.; Soldati, A. (2010). Orientation, distribution, and deposition of elongated, inertial fibers in turbulent channel flow, Physics of Fluids, Vol. 22, No. 3, 033301, doi:10.1063/1.3328874

[7] Ravnik, J.; Skerget, L.; Hribersek, M.; Zunic, Z. (2008). Numerical simulation of dilute particle laden flows by wavelet BEM-FEM, Computer Methods in Applied Mechanics and Engineering, Vol. 197, No. 6-8, 789-805, doi:10.1016/j.cma.2007.09.007

[8] Ravnik, J.; Skerget, L.; Zunic, Z. (2009). Combined single domain and subdomain BEM for 3D laminar viscous flow, Engineering Analysis with Boundary Elements, Vol. 33, No. 3, 420-424, doi:10.1016/j.enganabound.2008.06.006

[9] Maxey, M. R.; Corrsin, S. (1986). Gravitational settling of aerosol particles in randomly oriented cellular flow fields, Journal of the Atmospheric Sciences, Vol. 43, No. 11, 1112-1134, doi:10.1175/1520-0469(1986)043<1112:GSOAPI $>2.0 . \mathrm{CO} ; 2$

[10] Maxey, M. R. (1987). The motion of small spherical particles in a cellular flow field, Physics of Fluids, Vol. 30, No. 7, 1915-1928, doi:10.1063/1.866206

[11] Rubin, J.; Jones, C. K. R. T.; Maxey, M. (1995). Settling and asymptotic motion of aerosol particles in a cellular flow field, Journal of Nonlinear Science, Vol. 5, No. 4, 337-358, doi:10.1007/BF01275644

[12] Fung, J. C. H. (1997). Gravitational settling of small spherical particles in unsteady cellular flow fields, Journal of Aerosol Science, Vol. 28, No. 5, 753-787, doi:10.1016/S0021-8502(96)00478-8

[13] Chan, C. C.; Fung, J. C. H. (1999). The change in settling velocity of inertial particles in cellular flow, Fluid Dynamics Research, Vol. 25, No. 5, 257-273, doi:10.1016/S0169-5983(98)00042-2

[14] Marchioli, C.; Fantoni, M.; Soldati, A. (2007). Influence of added mass on anomalous high rise velocity of light particles in cellular flow field: A note on the paper of Maxey (1987), Physics of Fluids, Vol. 19, No. 9, 098101, doi:10.1063/1.2759528

[15] Tallapragada, P.; Ross, S. D. (2008). Particle segregation by Stokes number for small neutrally buoyant spheres in a fluid, Physical Review E, Vol. 78, No. 3, 9 pages (036308), doi:10.1103/PhysRevE.78.036308

[16] Maxey, M. R.; Riley, J. J. (1983). Equation of motion for a small rigid sphere in a nonuniform flow, Physics of Fluids, Vol. 26, No. 4, 883-889, doi:10.1063/1.864230

[17] Sobral, Y. D.; Cunha, F. R. (2003). A stability analysis of a magnetic fluidized bed, Journal of Magnetism and Magnetic Materials, Vol. 258-259, 464-467, doi:10.1016/S0304-8853(02)01098$\underline{3}$

[18] Hong, R. Y.; Ren, Z. Q.; Han, Y. P.; Li, H. Z.; Zheng, Y.; Ding, J. (2007). Rheological properties of water-based $\mathrm{Fe}_{3} \mathrm{O}_{4}$ ferrofluids, Chemical Engineering Science, Vol. 62, No. 21, 5912-5924, doi:10.1016/j.ces.2007.06.010 